\title{
ON THE METHEMOGLOBIN FORMATION BY AROMATIC NITRO AND AMINO COMPOUNDS
}

\author{
METHEMOGLOBIN FORMATION BY AMINOPHENOL \\ AND DIPHENOL IN VITRO \\ Taichi NAKAJIMA and Shigeko KUSUMOTO \\ Osaka Public Healh Institute, Morimachi, Higashinari-ku, Osaka
}

(Received May 24, 1963)

\begin{abstract}
For the study on the mechanism of methemoglobinaemia in the industrial poisoning due to the aromatic nitro and amino derivatives, AP and DP, were used in our study, because they are the main intermediates in the organism. The authors have found previously that. metHb was formed in the red blood cell by incubating it with AP or DP, and that, furthermore, oxygen uptake was occured during the incubation. Lately, it was proved that oxygen uptake was truly caused by the interaction between $A P$ or $D P$ and $H b$ in the red cell. Accordingly, the following study was made to elucidate the mechanism of the metHb forming reaction of $\mathrm{AP}$ or $\mathrm{DP}$ by using crystallized $\mathrm{Hb}$. In this paper, the following results are reported. MetHb was formed by ortho and para isomers of AP or DP. Those were the same as the substances which were previously reported to produce their quinone bodies autoxidatively or catalytically by the $\mathrm{Hb}$. It was suggested that the mechanism of metHb formation by AP or DP was different from by other popular metHb formers such as ferricyanide and nitrite. Unexpectedly, the reducing action of AP or DP was found when added into the $\mathrm{Hb}$ solution containing high amount of metHb. Moreover, as the perturbation of $\mathrm{Hb}$ in the reaction mixture was proved in this study, the mechanism of metHb formation by AP or DP is concluded as follows. The quinone bodies of AP or DP are produced by the oxidase action of $\mathrm{Hb}$ perturbed by AP or DP, then, those quinones act on $\mathrm{Hb}$ molecule and form metHb.
\end{abstract}

As one of the methemoglobin formers, quinone bodies of aminophenol and diphenol were formerly reported by Heubner ${ }^{1}$. The authors also suggested recently the catalytic quinone formation by hemoglobin as one of the cause of the industrial methemoglobinaemia due to the aromatic nitro and amino compounds ${ }^{2,3}$. This paper deals

Abbreviations: Hemoglobin; Hb, Methemoglobin ; metHb, Diphenol; DP, Aminophenol ; AP, Hydroquinone; Hydq., Pyrocatechine; PCC, o-Aminophenol; oAP, p-Aminophenol ; pAP, mAminophenol ; mAP, p-Benzoquinone; pBq. 


\section{METHEMOGLOBIN FORMATION}

with the mechanism of methemoglobin formation by aminophenol and diphenol.

\section{MATERIALs AND Methods}

Materials....... The red cell suspension: The red blood cells of normal adults were

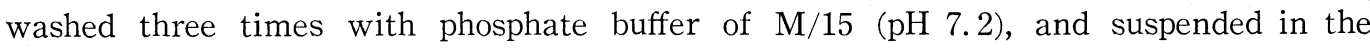
same buffer solution. The hemolysate: Washed red cells were lysed with a diluted phosphate buffer of $\mathrm{M} / 60$ ( $\mathrm{pH} 7.2$ ), and then centrifuged at $1300 \times \mathrm{g}$ for $10 \mathrm{~min}$.

Preparation of Human Hemog'obin........ Crystallized $\mathrm{Hb}$ was obtained from citrated adult human blood according to the Drabkin's method ${ }^{4}$.

Quantitative Determination of Methemoglobin........ The method of Evelyn and Malloy ${ }^{5}$ was modified partially. Sample was centrifuged at $1300 \times \mathrm{g}$ for $10 \mathrm{~min}$. Two milliliters of the supernatant were diluted with $7 \mathrm{ml}$ of $\mathrm{M} / 60$ phosphate buffer ( $\mathrm{pH}$ 6.6), and then divided into two cubetts $(1 \times 1 \times 4 \mathrm{~cm})$. One was added a few drops of neutralized natrium cyanide solution of $5 \%$ and the other the equal drops of phosphate buffer of $\mathrm{M} / 60$ ( $\mathrm{pH}$ 6.6). The amount of metHb was determined by measuring the difference of the optical density at $630 \mathrm{~m} \mu$ between them.

Quantitative Determination of Total Hemog'obin.........Following two methods were used. a) Cyan-metHb method ${ }^{5,6)} ; 0.5$ milliliters of the supernatant were diluted with $7 \mathrm{ml}$ of $\mathrm{M} / 60$ phosphate buffer $(\mathrm{pH} 6.6$ ) and then added a few drops of potassium ferricyanide (20\%). After standing for $10 \mathrm{~min}$ at room temperature, a few drops of neutralized sodium cyanide (5\%) were added. The amount of total $\mathrm{Hb}$ was determined by measuring the optical density at $540 \mathrm{~m} \mu$. b) Reduced-Hb method ${ }^{7)} ; 0.5$ milliliters of the supernatant were diluted with natrium carbonate $(0.1 \%)$. Sealing with petrol immediately after addition of a microspartula of hydrosulfite, measured the optical density at $556 \mathrm{~m} \mu$ and estimated the total amount of $\mathrm{Hb}$.

Determination of Millimolar Extinction Coefficient........ $\varepsilon_{m M}^{630 m \mu}=11.20$ was obtained by using Acuglobin (Ortho Pharmaceutical Corp. U. S. A.). $\varepsilon_{m M}^{556 m \mu}=13.08$ and $\varepsilon_{m M}^{830 m^{\mu}}=3.13$ were estimated using normal adult hemolysate of those total $\mathrm{Hb}$ was already determined by cyan-metHb method. (Confidence limit ; $\pm 2.7 \%$ )

Experimental Procedure........Buffered $\mathrm{Hb}$ preparations were incubated with AP, DP or other popular metHb formers at $38^{\circ} \mathrm{C}$ for $3 \sim 4$ hours. The initial concentration of $\mathrm{Hb}$ and agents were respectively ca. $0.3 \mathrm{mM}$ and $5 \mathrm{mM}$. At adequate intervals, an aliquot was pipetted out from the reaction mixture and it was cleared by centrifuging at $1300 \times \mathrm{g}$ for $10 \mathrm{~min}$. The amount of metHb and total $\mathrm{Hb}$ in the supernatant were determined by the methods described above.

When red cell suspension was incubated with metHb former, the cells, which were pipetted out from the mixture, were lysed by adding phosphate buffer of $\mathrm{M} / 60$ ( $\mathrm{pH}$ 6.6). After centrifuging, the amount of metHb and total $\mathrm{Hb}$ in the clear supernatant were estimated. 


\section{T. NAKAJIMA AND S. KUSUMOTO}

\section{RESULTS}

On the Methemoglobin Formation........When hemolysate (ca. $0.3 \mathrm{mM}$ ) was incubated with metHb former such as $\mathrm{AP}, \mathrm{DP}$ or $\mathrm{pBq}(5 \mathrm{mM})$ at $\mathrm{pH} 7.2(\mathrm{M} / 15)$ and $38^{\circ} \mathrm{C}$, a large amount of metHb was formed at a high rate. The results are shown in Fig. 1. As seen from Fig. 1, ortho and para isomers of AP and DP were effective as the metHb former, while meta isomers were not. The amount of metHb formed by the action of AP or DP reached to the maximum, which was about a half of the initial concentration of $\mathrm{Hb}$, at about one hour after the beginning of the reaction. Thereafter, the amount of metHb formed decreased slightly. When $\mathrm{pBq}$ was used as the metHb former, the rate of metHb formation was much larger than the rate obtained by using AP or DP. The maximum amount of metHb was obtained within 10 minutes

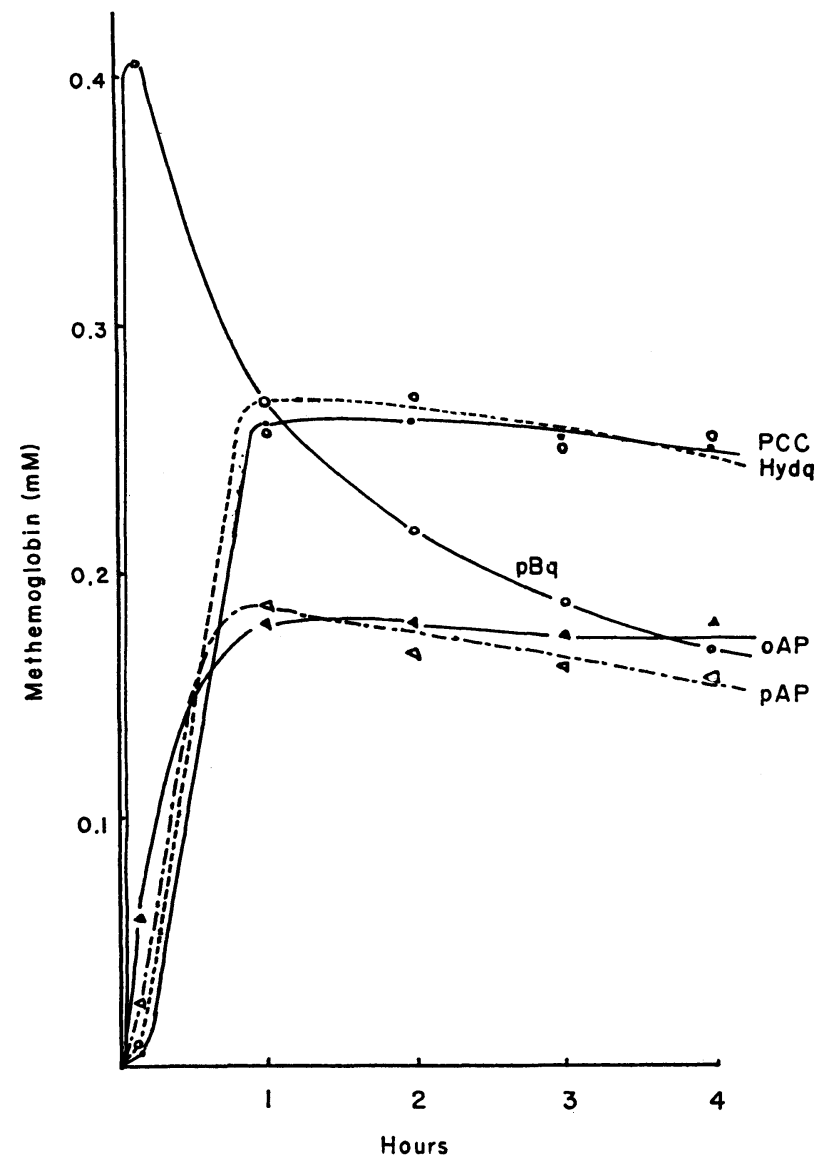

Fig. 1. MetHb formation in the hemolysate incubated with $\mathrm{AP}, \mathrm{DP}$ and $\mathrm{pBq}$. Initial concentration of the reagent; $5 \mathrm{mM}$. Initial concentration of total $\mathrm{Hb}$; $0.38 \mathrm{mM}$ when AP or DP was used and $0.42 \mathrm{mM}$ when $\mathrm{pBq}$ was used. Experiments were carried out at $\mathrm{pH} 7.2$ (phosphate buffer of $\mathrm{M} / 15$ ) and $38^{\circ} \mathrm{C}$. 


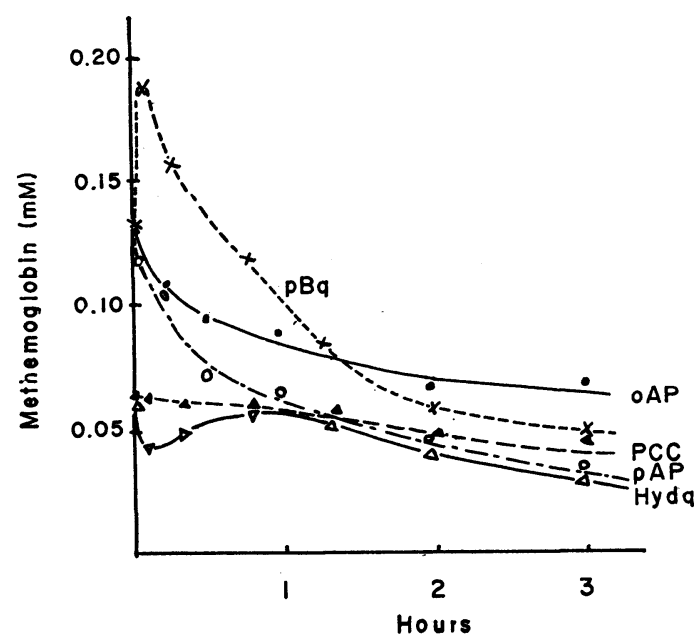

Fig. 2. The reduction of metHb by $\mathrm{AP}, \mathrm{DP}$ or $\mathrm{pBq}$.

Crystallized $\mathrm{Hb}$ used in these experiments contained $60 \sim 70 \%$ of metHb which was formed by standing hemoglobin in crystallized state for several weeks in a cold room of $2{ }^{\circ} \mathrm{C}$. Initial concentration of the reagents; $5 \mathrm{mM}$. Initial cocentration of total $\mathrm{Hb} ; 0.1 \sim 0.2 \mathrm{mM}$. Experiments were made at $\mathrm{pH} 7.2$ (M/15 phosphate buffer) and $38^{\circ} \mathrm{C}$.

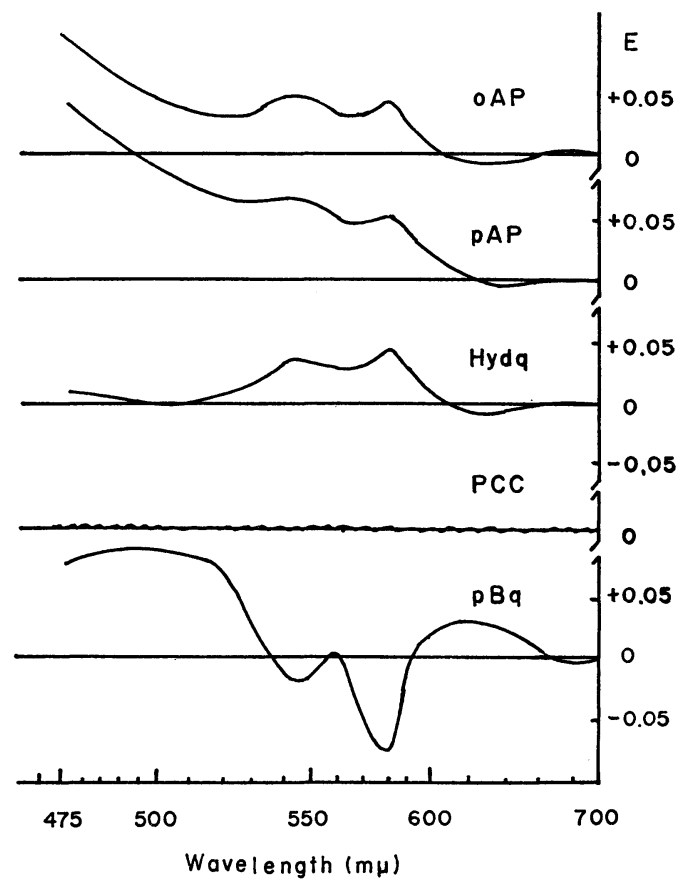

Fig. 3. The difference spectra of crystallized $\mathrm{Hb}$ solution incubated with AP, DP and $\mathrm{pBq}$ for $20 \mathrm{~min}$ at $27^{\circ} \mathrm{C}(\mathrm{pH} 6.8, \mathrm{M} / 15$ phosphate buffer).

Measurement of the difference spectrum was made by using Beckman Recording Spectrophotometer. Initial concentration of the reagents; $1 \mathrm{mM}$. The $\mathrm{Hb}$ solution used here was the same as that used in the experiments of Fig. 2. A strong light absorbance in the range of shorter wavelength may be due to that of the reagent. 
after the beginning of the reaction and the amount was about $90 \%$ of the initial concentration of $\mathrm{Hb}$. Then, it decreased rapidly. The same results were also obtained by using the red cell suspension or crystallized $\mathrm{Hb}$ solution.

The decrease of the amount of metHb formed by the action of $\mathrm{AP}, \mathrm{DP}$ or $\mathrm{pBq}$, which was observed in the later state of the incubation, was ascertained by the following experiments. When AP or DP was introduced to the crystallized $\mathrm{Hb}$ solution, which contained $60 \sim 70 \%$ of metHb, the metHb content decreased as shown in Fig. 2 . The results shown in Fig. 2 corresponded well to those shown in Fig. 3. Fig. 3 shows the difference spectrum between $\mathrm{Hb}$ solution (metHb content, 60 70\%) at zero time and that at 20 minutes after mixing the $\mathrm{Hb}$ solution with the metHb former, where the increase of optical densities at 580 and $540 \mathrm{~m} \mu$ shows the increase of the amount of oxyHb and the decrease of metHb. From these experiments, it was cleared that metHb was reduced to $\mathrm{oxyHb}$ by $\mathrm{AP}$ or $\mathrm{DP}$, and $\mathrm{PCC}$ was not effective for the reduction of metHb.

On the Total Amount of Hemoglobin......... By incubating Hb solution with AP or DP, the fibrous precipitates appeared. The precipitates seemed presumably to be proteinous because it was positive for protein-tests. As it was assumed that this fibrous precipitate was the denatured product of $\mathrm{Hb}$, the total amount of $\mathrm{Hb}$ in the incubated mixture was determined. The cyan-metHb method and the reduced-Hb method were used for the quantitative determination of total $\mathrm{Hb}$. The results are shown in Fig. 4. As shown in Fig. 4, the total $\mathrm{Hb}$ value measured by the reduced-Hb method decreased

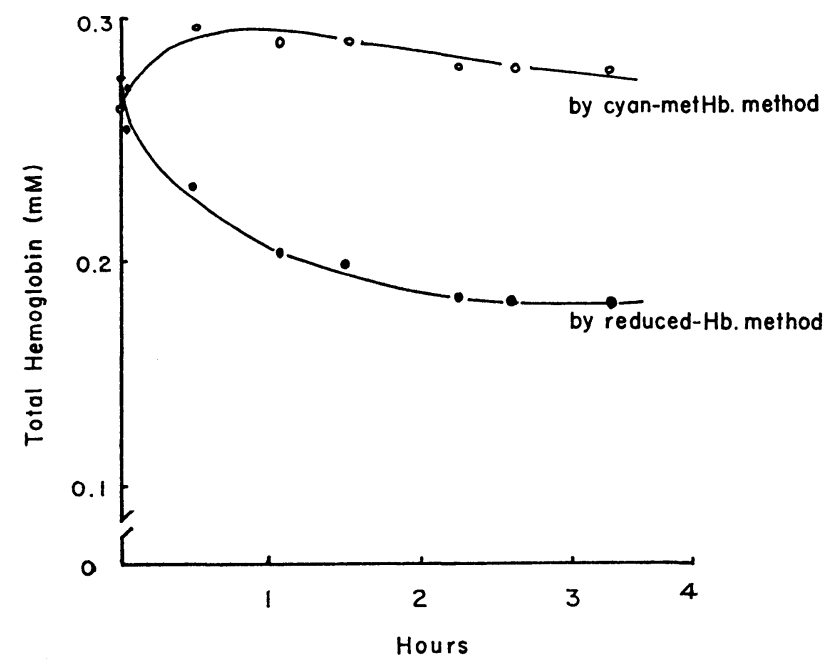

Fig. 4. The changes of the amounts of total $\mathrm{Hb}$ in the hemolysate incubated with pAP.

Initial concentration of pAP ; $5 \mathrm{mM}$. Initial concentration of $\mathrm{Hb} ; 0.3 \mathrm{mM} . \circ-\circ$; by the cyan-metHb method, - — - ; by the reduced-Hb method. Experimental condition; $\mathrm{pH} 7.2\left(\mathrm{M} / 15\right.$ phosphate buffer) and $38^{\circ} \mathrm{C}$. 


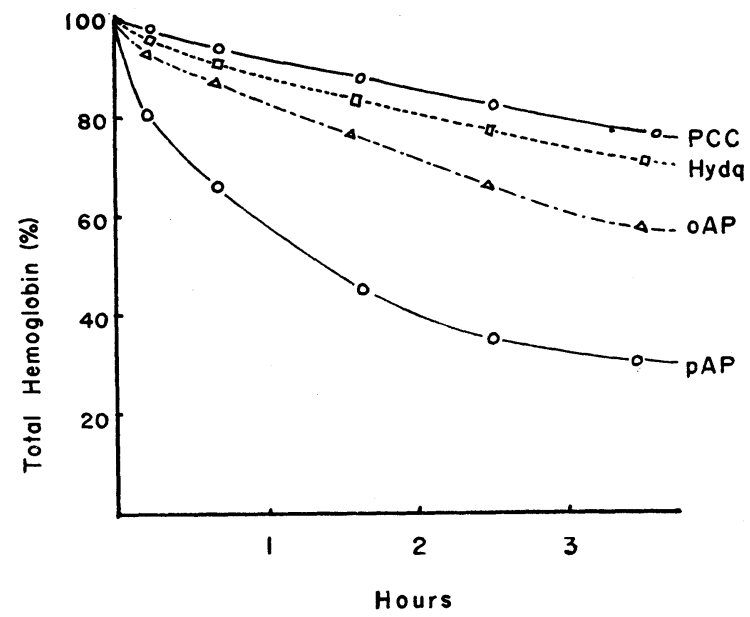

Fig. 5. Decreasing rate of total $\mathrm{Hb}$ in the crystallized $\mathrm{Hb}$ solution incubated with AP or DP.

The amounts of total $\mathrm{Hb}$ was represented as percentage to the initial $\mathrm{Hb}$ concentration. Procedures were described in Fig. 4.

markedly, whereas the value measured by the cyan-metHb method did not. The disagreement of total $\mathrm{Hb}$ values measured by the two different methods was found out when AP or DP was used, but not when meta isomers of them and $\mathrm{pBq}$ were used. The decreasing rate of the amount of total $\mathrm{Hb}$ measured by the reduced- $\mathrm{Hb}$ method was the largest in the case of pAP, and followed in the order of oAP, Hydq. and PCC (Fig. 5). This order was the same with the experiments using the other $\mathrm{Hb}$ preparations. Among them, the most remarkable decrease was shown in the crystallized $\mathrm{Hb}$ solution.

\section{DiscUsSION}

As described in the results, agents which formed metHb were para and ortho isomers of $\mathrm{AP}$ and $\mathrm{DP}$, and these were the substrates of oxidase action of $\mathrm{Hb}$, or the substances being autoxidative as reported previously. The meta isomers of AP and DP could not form metHb. It was already known that the meta isomers of AP and DP were not autoxidative and also not oxidizable by $\mathrm{Hb}$. Therefore, metHb formation was regarded as the oxidation of $\mathrm{Hb}$ by quinone bodies produced by the oxidation of AP and DP. These facts supported well Heubner's opinion ${ }^{1)}$. MetHb was formed remarkably in $\mathrm{pBq}$. The reaction of metHb formation by $\mathrm{pBq}$ was very fast as by the known popular metHb formers such as ferricyanide and nitrite. The fast metHb formation by those metHb formers may be due to the primary oxidation of heme iron without the action on the globin part. On the contrary, slow rate of metHb formation in AP or DP was similar to that of the reaction in which oxygen was uptaken during the incubation of $\mathrm{AP}$ or $\mathrm{DP}$ with or without $\mathrm{Hb}^{2 \sim 3}$. The gradual metHb formation, accordingly, may be due to the secondary oxidation of heme iron by the oxidized 


\section{T. NAKAJIMA AND S. KUSUMOTO}

forms of $\mathrm{AP}$ or $\mathrm{DP}$, or due to the perturbation of $\mathrm{Hb}$ by $\mathrm{AP}$ or $\mathrm{DP}$.

It was interesting that the amount of metHb decreased immediately after the addition of AP or DP to the $\mathrm{Hb}$ solution containing initially the high amount of met$\mathrm{Hb}$. As the decrease of metHb corresponded with the increase of oxyHb in the difference spectra, the decrease of metHb would be caused by the reduction of it. Probably, $\mathrm{AP}$ or DP acts on metHb as the reducing agent in such a case that the initial concentration of metHb was high. The reducing action of AP and DP seemed to be interpreted from the level of the redox potential which depended upon the proportion of oxy- and metHb, because the reduction was not observed when the initial concentration of metHb was low.

When Hydq. was added to the $\mathrm{Hb}$ solution containing initially the high amount of metHb, the change of total $\mathrm{Hb}$ in Hydq. was particularly different in its time course from that of AP or PCC. It is not yet understood why metHb temporarily reincreased after the initial decrease only in the case of Hydq.

For the cause of disagreement of total $\mathrm{Hb}$ values determined by the two methods, the cyan-metHb method and the reduced-Hb method, authors would like to speculate as follows. If it were correct to assume that the denatured $\mathrm{Hb}$ would be splitted to heme and globin, approximate constancy of maximum level of the amount of total $\mathrm{Hb}$ determined by the cyan-metHb method might be resulted from the artificial formation of dicyan-hematin, because the absorption band and its optical density of dicyanhematin were very similar to those of cyan-metHb. On the other hand, the decrease of total $\mathrm{Hb}$ by the reduced- $\mathrm{Hb}$ method might be resulted from the increase of splitted free heme having the lower optical density than reduced $\mathrm{Hb}$ at $556 \mathrm{~m} \mu$. Those assumptions are under the experimental study. Anyway, AP and DP, except their meta isomers, seemed to act on $\mathrm{Hb}$ as the perturbator similary as salicylate or benzoate, which were reported as the perturbator for $\mathrm{Hb}$ in the study using $\mathrm{Hb}$-ascorbic acid- $\mathrm{O}_{2}$ system by G. Kikuchi and T. Tomimura ${ }^{8}$. In our other experiment, hemichrome was detectable in the incubation mixture at the lower $\mathrm{pH}$ than in the native metHb solution. This fact shows that the perturbation of $\mathrm{Hb}$ might be sure to occur also in these experiments. Accordingly, oxidase action of $\mathrm{Hb}$ incubated with $\mathrm{AP}$ or DP would be understood as the varied function of the perturbed hemoglobin. After all, in the experiment using AP- or DP-Hb-O $\mathrm{O}_{2}$ system reported here, AP or DP seemed to be the perturbator as well as the oxidizable substrate. In meta isomers or in so-called primary metHb formers (ferricyanide, nitrite and $\mathrm{pBq}$ ), proteinous precipitates did not appeared ${ }^{9}$, and any findings to show the perturbing action were not found.

At present, the mechanism of metHb formation by AP or DP is supposed as follows. AP or DP is firstly oxidized to their quinone bodies by autoxidation or by the oxidase action of $\mathrm{Hb}$ perturbed by $\mathrm{AP}$ or $\mathrm{DP}$. Then, those quinone bodies act on $\mathrm{Hb}$ molecule and form metHb. Of course, metHb formed by quinone bodies might contain the secondarily formed metHb caused by perturbation of the globin part. From 


\section{METHEMOGLOBIN FORMATION}

these points of view, it is well explained why meta isomers can not form metHb.

\section{ACKNOWLEDGEMENT}

The authors wish to thank Dr. T. Okazaki (Japan Medical University, Tokyo) for the useful suggestions, particularly for the possible formation of dicyan-hematin, and also Dr. S. Kazihara (Director, Osaka Public Health Institute) for his helpful advice.

\section{REFERENCES}

1) Heubner, H. (1913). Arch. Exptl. Pathol. Pharmakol., 72, 241.

2) Nakajima, T. and Kusumoto, S. (1961). Quart. J. Labor Res., 9, 25.

3) Nakajima, T. and Kusumoto, S. (1961). Quart. J. Labor Res., 9, 29.

4) Drabkin, D. L. (1949). Arch. Biochem., 21, 224.

5) Evelyn, K. A. (1938). J. Biol. Chem. 126, 655.

6) Hashimoto, T. (1956). Japan. J. Nations Health, 27, 385.

7) Hunter, F. T. (1951). The Quantitation of Mixture of Hemoglobin Derivatives by Photoelectric Spectrophotometry (Edited by Hunter, F. T.), p. 112, Charles \& Thomas Co., Springfield.

8) Kikuchi, G. and Tomimura, T. (1954). J. Biochem. (Tokyo), 503, 41.

9) Kusumoto, S. and Nakajima, T. unpublished. 